\title{
COMMENT AND REVIEWS
}

\section{INBREEDING WHEN HOMOZYGOTES ARE AT A DISADVANTAGE : A REPLY}

\author{
B. I. HAYMAN and KENNETH MATHER \\ Agricultural Research Council's Unit of Biometrical Genetics, \\ Department of Genetics, University of Birmingham
}

In an earlier account (I953) we have considered progress towards homozygosis under inbreeding when homozygotes are at a disadvantage, taking into account a number of mating systems and types of selection. In disagreeing with our analysis Haldane (1956) makes two criticisms : first, that the equations are such as cannot be solved by the matrix methods we used ; and second, that our " principal results appear to be incorrect" (p. $\left.5^{6}\right)$. His paper is confined to a treatment of inbreeding under selfing. So in dealing with the points he raised we too will consider only this one mating system from among the number we discussed in our earlier paper.

Haldane's first criticism must spring from the mistaken belief that because the equations are non-linear they cannot be solved by matrix methods (p. 62). Now the relation between the proportions of genotypes in successive generations is linear apart from a variable factor of proportionality. It is well-known mathematically that the factor of proportionality can be ignored in such a case and the equations solved by matrix methods, provided the factor can be obtained as required from an independent source. Since the frequencies of genotypes in the population must sum to unity we clearly have such an independent source so that matrix methods are fully applicable.

More specifically, Haldane claims (p.62) that a statement on our p. 168 is equivalent to the false equations

$$
\begin{aligned}
& p_{1}=x p_{0}+\frac{1}{2} x q_{0} \\
& q_{1}=\frac{1}{2} q_{0}
\end{aligned}
$$

This claim is of course unwarranted : that these are in fact statements of proportionality is made clear by the footnote and the general formulæ for $p_{n}$ and $q_{n}$ given on the same page.

Turning now from our methods to consider our results, we may observe that despite his general charge of incorrectness, Haldane records agreement with us in respect, for example, of so important a result as the critical value of $\frac{1}{2}$ for the relative disadvantage of homozygotes, and nowhere cites a specific example of disagreement. True, on p. 63 he cites us as giving only one equilibrium frequency of heterozygotes whereas he finds that three different equilibria may be reached according to circumstances, which he attributes to insufficient care in our specification of the conditions of selection. In fact a second of his three equilibrium frequencies is explicitly given in our section 5.1 and the remaining one is implicit in the general statements of our section 5.2. As to the specification of conditions of selection his case of "seed selection" is the same as our detailed investigation, our "constant numbers of progeny" is identical with his "selection within lines only" and our " differential survival rates " explicitly includes selection both within and between lines. Indeed by their agreement with 
ours, Haldane's rederivation of the equilibrium frequencies bear witness not only to the correctness of our findings, but also to the adequacy of our specification of the conditions of selection.

To demonstrate this point more amply, however, and to bring out at the same time the power of the matrix method, let us complete the investigation suggested in our section 5.2, using the method of our 3.1 and 4.1 .

The generation matrix is

\begin{tabular}{c|ccc} 
& $a a$ & $b b$ & $a b$ \\
\hline$a a$ & $z$ & $\cdot$ & $\frac{1}{4} x$ \\
$b b$ & $\cdot$ & $w$ & $\frac{1}{6} y$ \\
$a b$ & $\cdot$ & $\cdot$ & $\frac{1}{2}$
\end{tabular}

where $x, y$ and $\mathrm{I}$ are the relative survival rates of $a a, b b$ and $a b$ in segregating families while $z$ and $w$ are the relative survival rates of $a a$ and $b b$ in truebreeding families. If $\left(p_{n}, q_{n}, r_{n}\right)$ are the proportions of $a a, b b$ and $a b_{0}$ in the $n$th generation, this matrix is to be interpreted as $p_{n+1} \alpha z p_{n}+\frac{1}{4} x_{n} r_{n}$, etc. reading along the rows. The latent roots and (proportional) frequencies in corresponding equilibrium populations are

$$
\begin{array}{ll}
z & \mathrm{~V}=(\mathrm{I}, \mathrm{o}, \mathrm{o}) \\
w & \mathrm{~V}^{\prime}=(\mathrm{o}, \mathrm{I}, \mathrm{o}) \\
\frac{1}{2} & \mathrm{~V}^{\prime \prime}=(s, t, \mathrm{I})
\end{array}
$$

where $s=\frac{x}{2(1-2 z)}$ and $t=\frac{y}{2(1-2 w)}$ are compound survival parameters for each of the homozygotes.

When $z>\frac{1}{2}$, $w$ the population becomes homozygous aa.

When $w>\frac{1}{2}, z$ the population becomes homozygous $b b$.

(When $z=w>\frac{1}{2}$ the population becomes mixed homozygous $a a$ and $b b$.)

When $\frac{1}{2}>z, w$ heterozygosity persists and at equilibrium

$$
p=\frac{s}{s+t+\mathrm{I}}, q=\frac{t}{s+t+\mathrm{I}} \text { and } r=\frac{\mathrm{I}}{s+t+\mathrm{I}} .
$$

Haldane's three special cases are

(i) Selection within lines only. $z=w=\frac{1}{4} x+\frac{1}{4} y+\frac{1}{2}$. Then $z=w>\frac{1}{2}$ and heterozygotes disappear. This is the result in Haldane's equation (2) and our 5.1, although in the latter we had the equivalent restriction that $z=w=1$ and would have divided the third column of the matrix by its sum, $\frac{1}{4} x+\frac{1}{4} y+\frac{1}{2}$.

(ii) Selection between lines only. $x=y=1$. Haldane's equilibrium results are included in the above inequalities. In particular, if $\frac{1}{2}>z=w$

$$
r=\frac{\mathrm{I}-2 z}{2(\mathrm{I}-z)}
$$

(iii) Seed selection. $z=x$ and $w=y$. This is our section 4.1 , and Haldane's equations (12) are included. If further $x=y$ (our 2.1) then

$$
r=\frac{1-2 x}{1-x}
$$


It will be observed that generally the rate of approach to and the kind of equilibrium depend solely on the survival rates, $z$ and $w$, between lines. When heterozygosity persists, the equilibrium frequencies are determined by $s$ and $t$ so that quite different sets of values of $x, y, z$ and $w$ may lead to the same equilibrium. For instance, when the two homozygotes survive equally, one-third of the equilibrium population is heterozygous for survival rates within and between lines such as $\left(\frac{1}{2}, \frac{3}{8}\right),\left(1, \frac{1}{4}\right)$ and $(2,0)$.

Now since Haldane attributes the correctness of our equilibrium frequencies to certain special conditions we must also show that the population frequencies after $n$ generations of inbreeding can always be obtained from matrices as well as from difference equations.

We can express the initial frequencies $\left(p_{0}, q_{0}, r_{0}\right)$ in terms of $\mathrm{V}, \mathrm{V}^{\prime}$ and $\mathrm{V}^{n}$ which then increase at relative rates $z^{n}, w^{n}$ and $\frac{1}{2}^{n}$ as inbreeding progresses. Thus

$$
\left(p_{0}, q_{0}, r_{0}\right)=\left(p_{0}-s r_{0}\right) \mathrm{V}+\left(q_{0}-t r_{0}\right) \mathrm{V}^{\prime}+r_{0} \mathrm{~V}^{\prime \prime}
$$

and after $n$ generations of selfing

or

$$
\left(p_{n}, q_{n}, r_{n}\right) a z^{n}\left(p_{0}-s r_{0}\right) \mathrm{V}+w^{n}\left(q_{0}-t r_{0}\right)+2^{-n} r_{0} \mathrm{~V}^{n}
$$

$$
\begin{aligned}
& p_{n} a z^{n}\left(p_{0}-s r_{0}\right)+2^{-n} s r_{0} \\
& q_{n} \propto w^{n}\left(q_{0}-t r_{0}\right)+2^{-n} t r_{0} \\
& r_{n} a 2^{-n} r_{0} .
\end{aligned}
$$

Division of each of the quantities on the right-hand side by their sum converts these relations to equalities,

$$
\text { e.g. } \quad r_{n}=\frac{r_{0}}{(2 z)^{n}\left(p_{0}-s r_{0}\right)+(2 w)^{n}\left(q_{0}-t r_{0}\right)+(s+t+1) r_{0}} \text {. }
$$

Once again we consider Haldane's special cases.

(i) Selection within lines only. Then $s+t+\mathrm{I}=0$ and $r_{n}=(2 z)^{-n} r_{0}$. This is Haldane's equation (3) with a suitable change of notation.

(ii) Selection between lines only. We obtain Haldane's equations (7) and (10).

(iii) Seed selection. We obtain Haldane's equations (13), (14) and (15). This case, of course, follows directly from the matrix in our 4.1 and it is, therefore, pertinent to expand $r_{n}$ fully in terms of $x$ and $y$. Then

$$
r_{n}=\frac{2(\mathrm{I}-2 x)(\mathrm{I}-2 y) r_{0}}{(2 x)^{n}(\mathrm{I}-2 y)\left[2(\mathrm{I}-2 x) p_{0}-x r_{0}\right]+(2 y)^{n}(\mathrm{I}-2 x)\left[2(\mathrm{I}-2 y) q_{0}-y r_{0}\right]+}
$$

Placing $x=\mathrm{I}-k, y=\mathrm{I}-l$ and changing the other symbols appropriately to reproduce Haldane's notation gives his equation (I5).

We can drive home this point of identity between his and our results even further by supposing the two homozygotes to survive equally. We could then place $x=y$ in the previous form for $r_{n}$ to produce Haldane's equation ( 13 ) but instead let us derive it from the first and very elementary case on our p. 168. Formulæ are given there for differently defined $p_{n}$ and $q_{n}, q_{n}$ being the frequency of heterozygotes. (In the last term of $p_{n}$, $p x$ has been misprinted for $q x$.) On dividing the right-hand sides of these 
proportionalities by their sum and rearranging we find that the proportion of heterozygotes after $n$ generations is

$$
\frac{(1-2 x) q}{(1-x) q+(2 x)^{n}[1-2 x-(1-x) q]}
$$

where $q$ is the initial proportion of heterozygotes. This, with $1-x=k$ is Haldane's equation (13).

It appears to us, therefore, that far from showing us to be wrong, the results of Haldane's heavier treatment have been merely to confirm our earlier findings and in doing so to demonstrate not only the validity but also the mathematical economy of matrix methods in treating the problems of inbreeding under selfing. No doubt the series of papers which he appears to promise will do the same for the other mating systems we discussed in 1953. At the same time, however, we hope that this exposition will have cleared up any obscurities in our original paper and that it has helped towards a clearer understanding of this selection problem and our method of attacking it. Partly for this reason we have taken the opportunity of considering a case more general than those covered by our earlier detailed investigation and by Haldane's but we hope too that in doing so we have provided in terms of $s, t, z$ and $w$ a solution of the population frequencies after $n$ generations of selfing which will be both simple and useful.

Finally, we realise that the relative merits of two mathematical techniques can be of little import to the majority of geneticists who will be interested more in results than in the mathematical investigations behind them. It is important, however, that the geneticist should be able to place confidence in the results and formulæ which are offered for his use, so that a charge of faulty treatment and incorrect results is not lightly to be dismissed. We trust we have amply demonstrated that our findings can command the geneticist's confidence.

\section{REFERENCES}

HALDANE, J. B. S. 1956. The conflict between inbreeding and selection. fournal of Genetics, 54, 56-63.

haYman, B. I., AND MATHeR, K. I953. Progress of inbreeding when homozygotes are at a disadvantage. Heredity, 7, I65-183. 